\title{
Robust and Adaptive Integration of Multiple Range Images with Photometric Attributes
}

\author{
Ryusuke Sagawa Ko Nishino Katsushi Ikeuchi \\ Institute of Industrial Science, University of Tokyo
}

\begin{abstract}
Integration of multiple range images is important to make use of 3D data acquired from stereo systems, laser range finders, etc. We propose a new range image integration method based on volumetric representation. Unlike other volume-based integration methods, we adaptively subdivide voxels depending on the curvature of the surface to be reconstructed, providing efficient representation of the underlying geometry and efficient use of computational resources. In our range image merging framework, additional attributes, e.g., color, laser reflectance power, etc., can be taken into account as well as $3 D$ geometric information. This ability allows us to generate $3 D$ models preserving sharp edges around texture boundaries, thereby providing a good basis for efficient rendering and texture mapping. The overall framework is designed to be robust against noise, taking consensus carefully in both geometry and color, which could be suitable for $3 D$ model reconstruction from noisy stereo images. In this paper, we describe the system, and present several results of applying our framework to real data. We also present some other future applications based on our framework.

1. Introduction
\end{abstract}

When building a 3D model of a real world object, the problem of registration and integration (alignment and merging) of multiple range images becomes crucial. As most range sensing systems, e.g., stereo, structured light, laser range finder, return range images obtained from particular viewing points, each output range image covers only a portion of the target object surface. Thus, all of the range images must be transformed into one common coordinate system, registered with one another and then stitched together to comprise one geometric model. Alignment of multiple range images is accomplished by recording each local coordinate system a priori, e.g., by using motion controlled stages, mounting range finders on robot arms and so on, or by establishing point correspondences and minimizing the total distance between those points, e.g., featurebased methods [3, 17], ICP-based methods [1, 2, 8, 9, 23], etc.

For merging multiple pre-aligned range images, several approaches have been proposed. Turk and Levoy [29] proposed a method to "zipper" two range images at a time, by first removing overlapping portions of the meshes, next clipping one mesh against another, and then re-triangulating the mesh on the boundary. Although this is an intuitive process to merge two range images, pairwise merging does not work when merging multiple range images. Given a number of range images overlapping each other, a merging procedure which extracts the isosurface is necessary. Merging methods that make use of volumetric, implicit-surface representation and the marching-cubes algorithm [20] are suitable for this purpose. Hoppe et al. [16] construct 3D surface models by applying the marching-cubes algorithm to a discrete, implicit-surface function generated from a set of range images. After inferring local surface approximations from clouds of points based on tangent plane estimations, local search is accomplished to compute the signed distance from each voxel to the surface of the point set. Curless and Levoy [6] enhances Hoppe's algorithm in a few significant points. However, none of these methods, including several others [13], compensate for noise or extraneous point data; the data is assumed to be part of the object and noise is assumed to be negligible. Each of these methods suffers from inaccuracy due to their integration strategy, e.g., integrating unrelated observations, and these accuracy problems will affect the result even when the data is noise-free. Wheeler et al. [30, 31] addressed these important problems by designing a consensus surface algorithm. The consensus surface algorithm attempts to justify the selection of observations used to produce the average by finding a quorum or consensus of locally coherent observations. This process successfully eliminates many troublesome effects of noise and extraneous surface observations, and also provides desirable results with noise-free data.

Based on Wheeler's algorithm to robustly integrate multiple range images, we propose a range image integration method which can construct 3D models with photometric attributes. Considering applications that utilize geometric models, for instance, 3D object recognition and localization tasks, it is desirable to construct 3D models with additional attributes such as color and intensity. With the additional information provided by photometric attributes, higher accuracy and robustness can be expected from those applications. By taking a consensus of appearance changes of the 
target object from multiple range images, we reconstruct the 3D model with appearance values attached per vertex, successfully discarding outliers due to noise produced in the image-capturing process. This algorithm, taking consensus of photometric attributes, can also be used to derive the rigid part of the appearance change on the object surface, providing a 3D model with Lambertian reflected light values under a static illumination environment.

In some sense, our method is analogous to "Voxel Coloring" $[5,19,25]$, where photometric consistency is used to carve a volume to reconstruct a geometric model with texture. Our framework goes the opposite way; we already have 3D information; from it, we construct a photometric consistent geometric model.

We also propose an algorithm to construct the 3D model in an efficient representation. By taking the surface curvature into account when splitting the voxels recursively in an octree manner, the resulting 3D surface will be subdivided more in high curvature areas and less in surface areas that are near planar. Thus, the resulting geometric model will require less triangular patches to represent the object surface. Furthermore, by taking the photometric attributes into account, we can construct 3D models that have higher detail in surface areas that contain significant variation of appearance, providing an efficient basis for texture-mapping and shading. This is similar to research on mesh model simplification algorithms based on surface

$[12,14,15]$, while we reconstruct a simplified 3D model through a range image merging process based on implicit surface representation. The simplification is done when splitting voxels recursively, enabling better preservation of topology and mass of the object compared with results of other volume based simplification methods [26, 27]. Frisken et al.[11] proposed adaptive sampling of distance field; however, their method does not produce a triangular mesh model. For converting the volumetric representation of the 3D model a to triangular-based mesh model, we propose an extended version of marching-cube algorithm; this version handle voxels in different resolutions.

The remainder of this paper is as follows. In Section 2 , we review Wheeler's volumetric merging algorithm. In Section 3, we propose an algorithm to construct 3D models with photometric attributes. Section 4 describes the algorithm to construct adaptive resolutional 3D models based on both surface curvature and photometric attributes. Experimental results of applying the proposed method on real data are provided in Section 5; finally, Section 6 concludes the paper.

\section{Volumetric Range Image Integration}

In this section, we review the range image integration algorithm proposed by Wheeler [30, 31], which we will use as our basis.

In Wheeler's algorithm, all range images are first stored

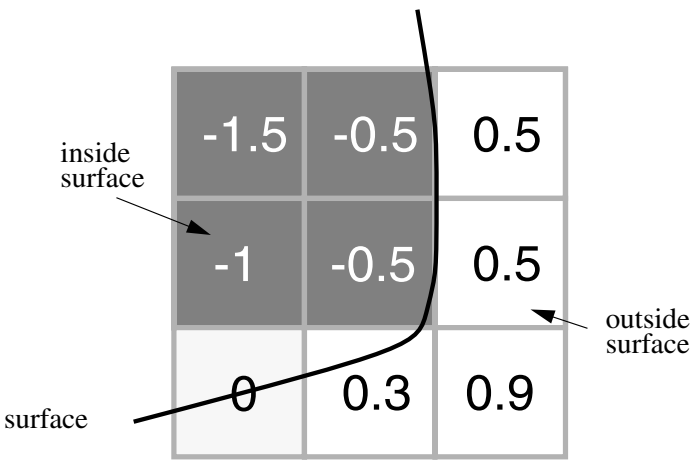

Figure 1: Zero-crossing interpolation from the grid sampling of an implicit surface

in a volumetric representation. The volume is split into grids, with each grid containing samples of an implicit surface. Namely, in each voxel, the signed distance $f(\boldsymbol{x})$ from the center point of the voxel $\boldsymbol{x}$ to the closest point of the object's surface. Positive values of $f(x)$ indicate that the voxel lies outside the surface, while negative values indicate that it lies inside. The range image integration (view merging) problem can be interpreted as, given multiple samplings of this implicit function through multiple range images, extract the isosurface that is the zero crossing of this function (Figure 1). To solve this problem, we first have to consider how to compute $f(\boldsymbol{x})$, without knowing which, of many possible surfaces, is the exact surface..

\subsection{Consensus Surface Algorithm}

Wheeler et al. proposed the consensus surface algorithm, to compute the signed distance function $f(\boldsymbol{x})$ for arbitrary points $\boldsymbol{x}$ when given $N$ triangulated surface patches from various views of the object surface.

Previous naive algorithms define the magnitude of the implicit function $|f(\boldsymbol{x})|$ of each voxel as the distance from the voxel center to the nearest triangle in all views (range images). However, this definition returns false values when range images contain errors. Figure 2 depicts this situation; the point chosen as the closest point from $\boldsymbol{x}$ does not belong to the real surface, and the naive algorithm incorrectly considers that $\boldsymbol{x}$ lies inside the object surface from noisy surface normal information.

This sensitivity to noise in range data can be solved by estimating the surface locally by averaging the observations of the same surface. Nearby observations are compared using their locations and surface normals, and if those values are within a predefined error tolerance, they can be considered to be observations of the same surface. The search for "nearby" observations can be accomplished using k-d trees [10] containing each range image separately. Given multiple surfaces that can be considered to be observations of the same surface, the consensus surface algorithm examines the closest point in each image's triangle set to determine whether it can contribute to the consensus, by check- 


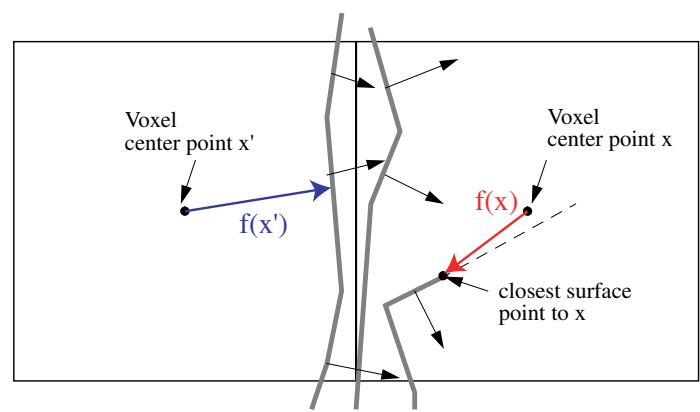

Figure 2: Naive algorithm: An example of inferring the incorrect sign of a voxel's value, $f(x)$, due to a single noisy triangle.

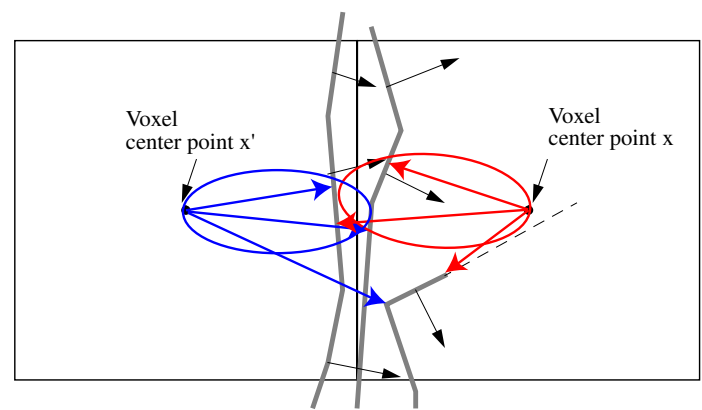

Figure 3: Consensus surface algorithm: The signed distance is chosen from the consensus surfaces inside the circle.

ing whether it is sufficiently close in terms of location and normal direction. A simple example when two range image surfaces are present is as follows.

$$
\begin{aligned}
& \text { SameSurface }\left(\left\langle\boldsymbol{p}_{0}, \boldsymbol{n}_{0}\right\rangle,\left\langle\boldsymbol{p}_{1}, \boldsymbol{n}_{1}\right\rangle\right)= \\
& \begin{cases}\text { True } & \left(\left\|\boldsymbol{p}_{0}-\boldsymbol{p}_{1}\right\| \leq \delta_{d}\right) \wedge\left(\boldsymbol{n}_{0} \cdot \boldsymbol{n}_{1} \geq \cos \theta_{n}\right) \\
\text { False } & \text { otherwise }\end{cases}
\end{aligned}
$$

where $\delta_{d}$ is the maximum allowed distance and $\theta_{n}$ is the maximum allowed difference in normal directions.

Range image surfaces that pass this check are considered to be the consensus surfaces, and the distance to the closest one of them is used as the signed distance (See Figure 3).

\subsection{Octree-based Voxel Splitting}

To represent surface in volume, the volume has to be fine only around where the surface lies, instead of having fixed resolution [6]. In Wheeler's algorithm, this is accomplished by recursively splitting the voxels in an octree manner. Voxels containing zero crossing or neighboring zero crossing implicit function values are subdivided into the finest level by checking the magnitude of the signed distance ${ }^{1}$. As the resolution of voxels containing the resulting surface are the same (Figure 4), the marching-cube algorithm [20] can be applied straightforwardly to extract the final triangularbased mesh surface.

\footnotetext{
${ }^{1}$ Refer to [30, 31] for details
}

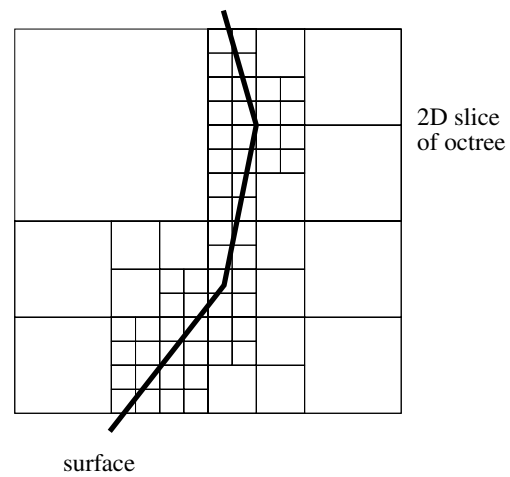

Figure 4: A 2D slice of the octree splitting volume. The resolution is high around the surface and low elsewhere.

\section{Consensus Surface with Photometric At- tributes}

In applications utilizing geometric models, e.g., 3D object recognition/localization, non-rigid appearance variation plays a crucial role in the accuracy and robustness. In particular, specular reflection causes the appearance to change non-rigidly, consequently making the whole process difficult. Thus, to date, most object recognition and object localization algorithms simply neglect specular reflection as outliers and assume Lambertian surfaces for target scene and model. To cooperate with this basic assumption on photometric properties, it is highly desirable to construct the geometric model to be used in such applications with Lambertian reflection property. If the 3D model is represented with photometric attributes that are rigid against changes in illumination and viewing directions, higher accuracy and robustness can be expected. Furthermore, if the illumination directions and viewing directions can be pre-estimated when processing recognition algorithms, non-rigid appearance variation such as specular reflection can be predicted and added to the 3D model appearance to further elevate the accuracy.

We accomplish this photometrically rigid 3D model construction in our range image integration framework. As examples of photometric attributes attached to range images, we consider two different attributes: laser reflectance strength and intensity/color.

In brief, laser range finders measure distance by shooting a laser and receiving its reflection from the target object. Distance to a particular point on the target object is computed by measuring the time duration between the laser shot and received back time in time-of-flight range finders, or by measuring the phase difference in phase-transition based range finders. In either case, the ratio of the discharged laser strength and the reflected laser strength can be returned per each 3D point. We will refer to this additional attribute of range images obtained from laser range finders as laser reflectance strength (LRS). As the laser can be considered 


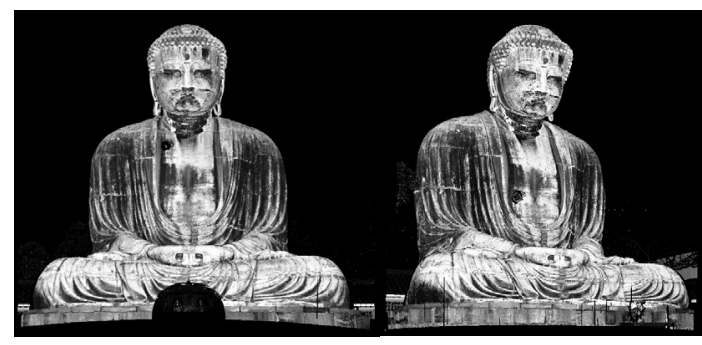

Figure 5: Two images of the Great Buddha in Kamakura, using LRS values as pixel values.

as light with very narrow wavelength distribution, almost a single value, the behavior of the reflected laser on the target surface can be considered as same as the general light reflection. Namely, almost isotropic reflection analogous to diffuse reflection and sharp reflection distributed around the perfect mirror direction analogous to specular reflection occurs. Since the laser reflected in the perfect mirror direction will not be observed from the range finder direction, the portion of the laser that is reflected back can be considered to be caused by this diffusive reflection. Figure 5 depicts two images using the LRS values attached to each 3D point as pixel values, rendered from the view point of the laser range finder. Although the LRS values are almost view-independent, they do vary slightly depending on the scanned direction [21].

To construct a 3D model with these LRS values attached to each vertex, we take the consensus of the LRS values from different range images as well as the 3D information when merging multiple range images. In this LRS attribute case, the consensus can be obtained simply by taking the median of the LRS values from multiple range images inside each voxel and assigning it to each voxel center.

As an example of applications utilizing this LRS value attached 3D model, robust 2D-3D registration can be considered [18].

The color or intensity of target objects can be handled in the same manner. Range images with color information attached per vertex can be obtained by using range finders that can acquire color images aligned to range images, e.g., Minolta Vivid900 etc., stereo systems, or by aligning 2D images taken separately to range images obtained by laser range finders $[18,28]$. As is well-known, the color variation on the object surface is composed of two reflection components: the diffuse reflection and the specular reflection. While the diffuse reflection is almost independent of the viewing direction and its strength varies depending on the illumination direction, the specular reflection changes its strength drastically depending on the viewing direction and illumination direction. For simplicity, we consider a situation where the color images of the target object are taken under a static illumination environment with only the viewing direction varying while the object stays static. This

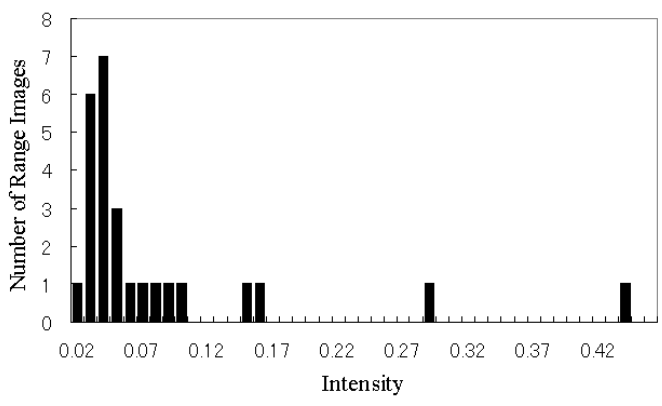

Figure 6: An example of the histogram of the intensity values of consensus points. Some outliers due to specular reflection are observed. In this case, the median value is 0.04 .

assumption can be made naturally when scanning objects with laser range finders, especially when the target object is large, and also when scanning small objects to build 3D models for use in object recognition tasks in indoor scenes. As the illumination direction can be considered to be static for all color images, the intensity variation of each $3 \mathrm{D}$ point on the target object surface should have a DC component because of the invariant diffuse reflection with a sharp peak caused by specular reflection added to it which can be observed from a narrow viewing direction. Thus, if each 3D point is observed from enough viewing directions, the histogram of the intensity values should have a sharp peak at the diffuse reflection value with some distribution around it due to image capturing noise. Figure 6 depicts an example of this from real data. Based on this consideration, by taking the the median from multiple range images inside each voxel and assigning it to each voxel, we can determine the color or intensity values to be attached to the resulting $3 \mathrm{D}$ model.

\section{Integrating in Adaptive Resolution}

The original consensus surface algorithm efficiently computes signed distances by utilizing an octree. However, it generated a mesh model in finest resolution everywhere. To reduce the amount of data to represent the object and to use computational resources efficiently, we propose a method which generates a mesh model in adaptive resolution; with appropriate resolution according to the geometric and photometric characteristics of the observed object.

\subsection{Voxel Subdivision based on Geometric At- tribute}

To efficiently represent the final mesh model, we determine the sampling interval of the signed distance depending on the variation of geometric attributes. As an example, we use the surface curvature. Depending on the change in surface curvature, the proposed method samples coarsely in planar areas, consequently reducing the amount of data and computation, while creating a finer model of an intricate object by utilizing the computation power efficiently.

Our method determines the variation of surface curvature 


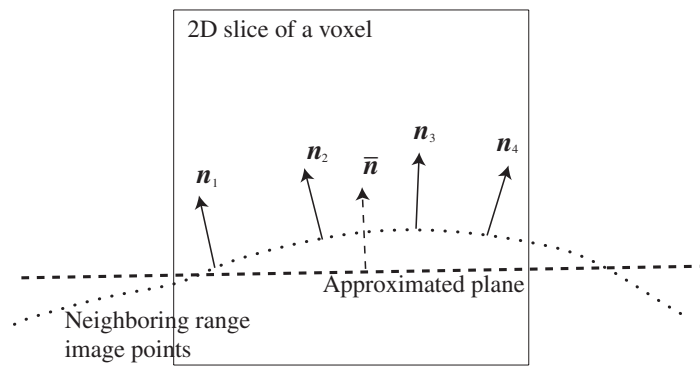

Figure 7: Our method approximates neighboring range images points to a plane and computes its normal vector $\bar{n}$ by principal component analysis (PCA) for the cloud of range image points. The approximate normal is then used to determine further subdivision.

comparing surface normals. We compare the normal $\boldsymbol{n}_{i}$ of each 3D point of all range images inside the voxel in interest and the normal $\overline{\boldsymbol{n}}$ of the approximated plane (See Figure 7 ), which can be estimated by applying PCA to all point data in the voxel. If the angle between data point normals $\boldsymbol{n}_{i}$ and approximate normal $\overline{\boldsymbol{n}}$ satisfies

$$
\max _{i}\left(\arccos \left(\boldsymbol{n}_{i} \cdot \overline{\boldsymbol{n}}\right)\right)<\delta_{n},
$$

where $\delta_{n}$ is the threshold of angle, the sampling interval is fine enough and no further voxel splitting is required.

To avoid erroneous subdivisions of voxels by the influence of noise included in each range image, our method takes a consensus between range images on decision of voxel subdivision. Now, $N_{n}$ is the number of range images which satisfies Eq.2. Our method does not subdivide the voxel if

$$
N_{n}>T_{n}
$$

where $T_{n}$ is the threshold of consensus for normal vectors.

\subsection{Voxel Subdivision based on Photometric At- tribute}

Voxel subdivision based on variation of photometric attributes can be accomplished in a similar manner. When the voxel is subdivided depending on geometric attributes only or without consideration of any attributes, the appearance of the resulting object will be significantly smoothed out. Since ordinary shaders such as smooth shading and phong shading will simply interpolate the intensity values attached to each vertex, this smoothing is unavoidable. However, if we can triangulate the $3 \mathrm{D}$ mesh model with regard to the appearance variation, i.e. fine around appearance boundaries and having each triangular patch contain almost the same texture color, simple shading will work dramatically well. Also, 3D models tessellated with regard to its texture variation are useful to accomplish further texture analysis and synthesis. For instance, view-dependent texture mapping like [22] can achieve higher compression, since global texture compression stacking triangular patches with similar texture can be applied.

In a similar manner to subdividing by the curvature of

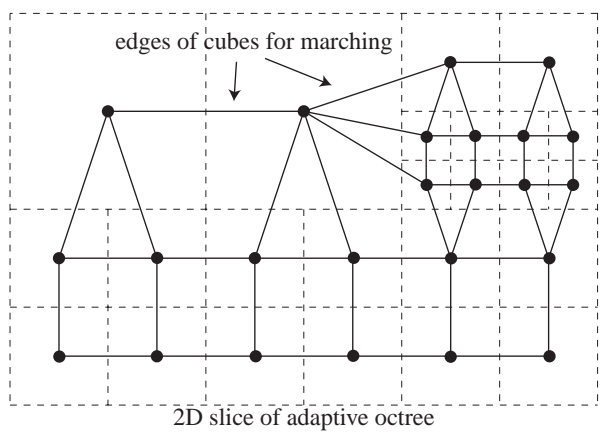

Figure 8: Edges connecting adjacent voxels in an adaptive octree: 3D space is partitioned into cubes or quadratic pyramids, etc. Marching cubes can be applied to these irregular forms without creating new tables of mesh generation for each form, because they are considered to be degenerated and transformed cubes.

the surface, our method computes the variation of photometric attributes of 3D points inside the voxel of interest. Now, $c_{i}, c_{j}$ are the photometric attributes of neighbor points included in a range image. If the maximum difference satisfies

$$
\max _{i, j}\left(\operatorname{Distance}\left(c_{i}, c_{j}\right)\right)<\delta_{c},
$$

where $\delta_{c}$ is the threshold and Distance $\left(c_{i}, c_{j}\right)$ is the function which computes the difference of two photometric attributes, the sampling interval is fine enough for the range image.

Our method also takes a consensus while considering photometric attributes. Similar to Eq.3, our method does not subdivide the voxel if

$$
N_{c}>T_{c},
$$

where $N_{c}$ is the number of range images which satisfies Eq.4 and $T_{n}$ is the threshold of consensus for photometric attributes.

\subsection{Marching Cubes for Adaptive Octree}

The original marching cubes algorithm can be applied only to voxels that have the same resolution (size). We extend the algorithm for triangulation of voxels in adaptive resolution generated from our method.

For voxels that are surrounded by voxels with the same resolution, the vertices of a cube to march are the central points of 8 adjacent voxels. In a similar manner, voxels surrounded by different size voxels will have a set of connected voxels in a form of quadratic pyramid or other special forms to march. Figure 8 shows the edges connecting adjacent voxels in an adaptive octree. Since these forms can be considered to be degenerated and transformed cubes, the original marching cube algorithm can be applied without modification.

\section{Experiments}

For this project, we have built a PC cluster that consists eight PCs, each equipped with dual PentiumIII $800 \mathrm{MHz}$ processors with $1 \mathrm{~GB}$ memory, connected by 100BASE-TX 
Table 1: Statistics of models of the Buddha: Our method reduces the amount of data and computation time. However, the mean errors are quite small compared to the Buddha size.

\begin{tabular}{|l|c|c|c|}
\hline & Number of points & Time for Integration & Mean Error \\
\hline (A) & 3.0 million & $252 \mathrm{~min}$. & N/A \\
\hline (B) & 1.2 million & $82 \mathrm{~min}$. & $0.99 \mathrm{~mm}$ \\
\hline (C) & 1.4 million & $88 \mathrm{~min}$. & $0.44 \mathrm{~mm}$ \\
\hline
\end{tabular}

Ethernet. As consensus surfaces can be computed independently requiring only adjacent voxels, we parallelize the whole process by splitting the whole volume into pieces. With this parallel implementation, we are able to handle a huge amount of range image data.

First, we observe the shape and intensity of a box using the light-stripe range finder [24]. We acquire 60 range and intensity images from various viewpoints by putting the box on a turntable and rotating it six degrees for each step. Since range and intensity images are already aligned, we can attach intensity values to corresponding 3D points as a photometric attribute. Specular reflection is observed in some images(See Figure 9).

Figure 10 shows the merging result of the box. We experimented with our algorithm in two cases: merging with adaptive subdivision based only on surface curvature and based on both surface curvature and intensity. As can be seen in Figure 10, although there are specular reflections in some input images, they are not observed in the resulting model.

When subdividing voxels only by the curvature of the surface, the sampling becomes coarse in the planar area. However, since the intensity varies drastically around the character edges, the characters are smoothed out and deformed when rendered with simple smooth shading. On the other hand, when voxel subdivision is accomplished based on both curvature and intensity, the consensus surface sampling becomes fine around the character edges. Because of this, the sharpness of intensity edges is preserved well and the shape of the characters are well rendered.

Next, we applied our algorithm to the Great Buddha of Kamakura, whose height is about $13 \mathrm{~m}$. Sixteen range images with LRS values attached to each 3D point were acquired by Cyrax 2400 [7]. Figure 11 shows 3 different results of our method. Their statistics are described in Table 1. We compared the size and accuracy of the models between $\mathrm{A}$ and $\mathrm{B}, \mathrm{A}$ and $\mathrm{C}$, using Metro [4].

In comparison of the images rendered with LRS values, the appearance of (B3) is smoothed out compared with (A3). On the other hand, the sharp edges due to variation in LRS values of (C3) are well preserved while successfully reducing the amount of the data.
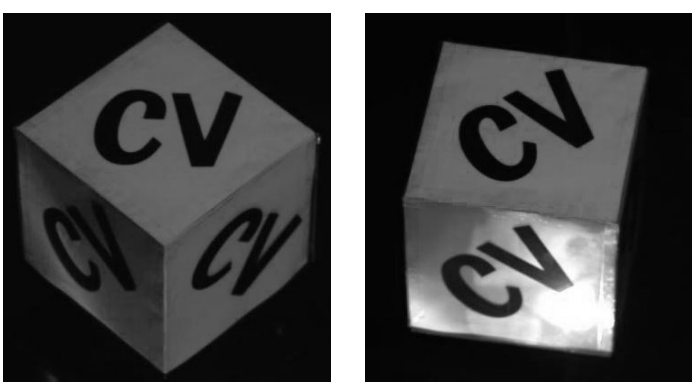

Figure 9: Range and intensity images of the box is acquired from various viewpoints using a turn table. These are 2 intensity images of 60 input images. Some images include specular reflection.

\section{Conclusion and Future Work}

In this paper, we proposed a range image integration framework which can construct 3D models with photometric attributes. By taking a consensus of appearance changes of the target object from multiple range images, we reconstruct the 3D model with an appearance which successfully discards outliers due to noise. Also, we can provide a model with Lambertian reflected light values by discarding specular reflection as outliers.

We also proposed an algorithm for constructing a 3D model in an efficient representation. Considering the surface curvature and the photometric attributes, we constructed 3D models that have higher detail in surface areas that contain either high curvature or significant variation of appearance. Thus, we could efficiently use computational resources.

For future work, we are considering several applications that make full use of models with photometric attributes, for instance 2D-3D registration, 3D localization, efficient texture mapping, global texture analysis, among others.

\section{References}

[1] R. Bergevin, M. Soucy, H. Gagnon, and D. Laurendeau. Towards a general multi-view registration technique. IEEE Trans. Patt. Anal. Machine Intell., 18(5):540-547, May 1996.

[2] P.J. Besl and N.D. McKay. A method for registration of 3-d shapes. IEEE Trans. Patt. Anal. Machine Intell., 14(2):239256, Feb 1992.

[3] Y. Chen and G. Medioni. Object modeling by registration of multiple range images. Image and Vision Computing, 10(3):145-155, Apr 1992.

[4] P. Cignoni, C. Rocchini, and R. Scopigno. Metro: measuring error on simplified surfaces. Computer Graphics Forum, 17(2):167-174, June 1998.

[5] W. Bruce Culbertson, Thomas Malzbender, and Greg Slabaugh. Generalized voxel coloring. In B. Triggs, A. Zisserman, and R. Szeliski, editors, Vision Algorithms: Theory and Practice, number 1883 in LNCS, pages 100-115. Springer-Verlag, sep 1999.

[6] Brian Curless and Marc Levoy. A volumetric method for building complex models from range images. In Proc. SIGGRAPH'96, pages 303-312. ACM, 1996.

[7] http://www.cyra.com. 

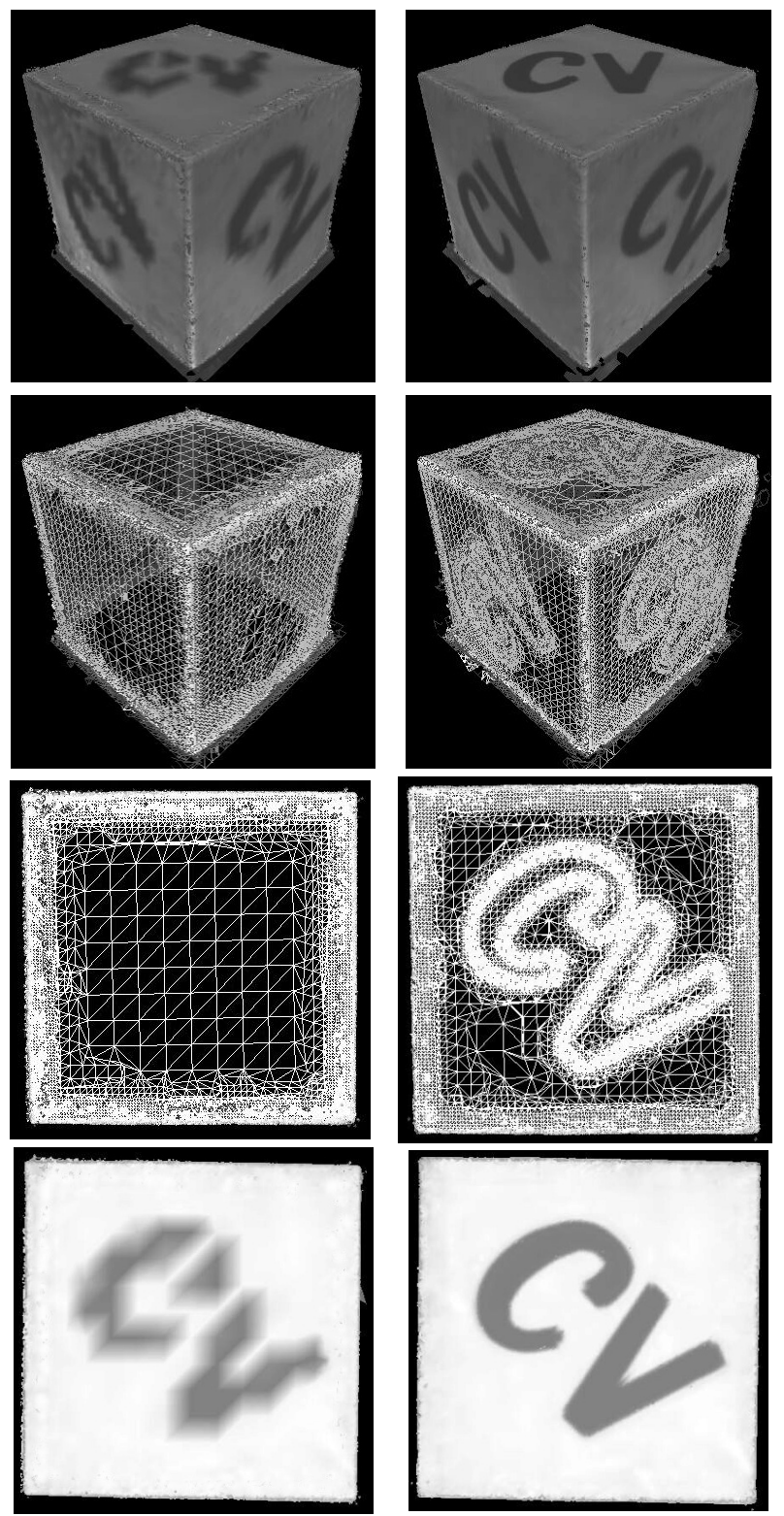

Figure 10: Merging results of a box with texture: the model in the left column is created by adaptive subdivision only based on the curvature of the surface, and the one in the right column is created by adaptive subdivision based on both the curvature and the variation of intensity.

[8] C. Dorai, G. Wang, A.K. Jain, and C. Mercer. From images to models: Automatic 3d object model construction from multiple views. In Proc. of the 13th IAPR International Conference on Pattern Recognition, pages 770-774, 1996.

[9] D.W. Eggert, A.W. Fitzgibbon, and R.B. Fisher. Simultaneous registration of multiple range views for use in reverse engineering. Technical Report 804, Dept. of Artificial Intelligence, University of Edinburgh, 1996.

[10] Jerome H. Friedman, Jon Bentley, and Raphael Finkel. An algorithm for finding best matches in logarithmic expected time. ACM Transactions on Mathematical Software, 3(3):209-226, 1977.
[11] S.F. Frisken, R.N. Perry, A.P. Rockwood, and T.R. Jones. Adaptively sampled distance fields: A general representation of shape for computer graphics. In Proc. SIGGRAPH200O, pages 249-254. ACM, July 2000.

[12] M. Garland and P.S. Heckbert. Simplifying surfaces with color and texture using quadric error metrics. In Proc. IEEE Visualization 1998, 1998.

[13] A. Hilton, A.J. Stoddart, J. Illingworth, and T. Windeatt. Reliable surface reconstruction from multiple range images. In Proceedings of European Conference on Computer Vision, pages 117-126, Springer-Verlag, 1996.

[14] H. Hoppe. Progressive meshes. In Computer Graphics (SIGGRAPH 1996 Proceedings), pages 99-108, 1996.

[15] H. Hoppe. New quadric metric for simplifying meshes with appearance attributes. In Proc. IEEE Visualization 1999, pages 59-66, 1999.

[16] H. Hoppe, T. DeRose, T. Duchamp, J.A. McDonald, and W. Stuetzle. Surface reconstruction from unorganized points. In Proc. SIGGRAPH'92, pages 71-78. ACM, 1992.

[17] A. Johnson and M. Hebert. Surface registration by matching oriented points. In Proc. Int. Conf. On Recent Advances in 3D Digital Imaging and Modeling, pages 121-128, May 1997.

[18] R. Kurazume, M.D. Wheeler, and K. Ikeuchi. Mapping textures on $3 \mathrm{~d}$ geometric model using reflectance image. In Proc. Workshop on Data Fusion Applications for Intelligent Systems, Int. Conf. on Robotics and Automation, 2001.

[19] K.N. Kutulakos and S.M. Seitz. What Do N Photographs Tell Us about 3D Shape? Technical Report 680, Computer Science Dept. U. Rochester, Jan. 1998.

[20] W. Lorensen and H. Cline. Marching cubes: a high resolution 3d surface construction algorithm. In Proc. SIGGRAPH'87, pages 163-170. ACM, 1987.

[21] S.K. Nayar and M. Oren. Generalization of the lambertian model and implications for machine vision. International Journal of Computer Vision, 14:227-251, 1995.

[22] K. Nishino, Y. Sato, and K. Ikeuchi. Eigen-texture method: Appearance compression based on $3 \mathrm{~d}$ model. In Proc. of Computer Vision and Pattern Recognition '99, volume 1, pages 618-624, Jun. 1999.

[23] K. Pulli. Multiview registration for large data sets. In Second Int. Conf. on 3D Digital Imaging and Modeling, pages 160168, Oct 1999.

[24] K. Sato and S. Inokuchi. Three-dimensional surface measurement by space encoding range imaging. Robotic Systems, 2(1):27-39, 1985.

[25] S.M. Seitz and C.R. Dyer. Photorealistic Scene Reconstruction by Voxel Coloring. In Proc. of Computer Vision and Pattern Recognition '97, pages 28-34, 1997.

[26] R. Shekhar, E. Fayyad, R. Yagel, and J. Cornhill. Octreebased decimation of marching cubes surfaces. In Proc. Visualization'96, pages 335-342, 1996.

[27] R. Shu, Z. Chen, and M.S. Kankanhalli. Adaptive marching cubes. The Visual Computer, 11:202-217, 1995.

[28] I. Stamos and P.K. Allen. Registration of 3d with 2d imagery in urban environments. In Proc. the Eighth International Conference on Computer Vision, to appear, Vancouver, Canada, 2001.

[29] G. Turk and M. Levoy. Zippered polygon meshes from range images. In SIGGRAPH 94, pages 311-318, Jul 1994.

[30] Mark D. Wheeler. Automatic Modeling and Localization for Object Recognition. PhD thesis, School of Computer Science, Carnegie Mellon University, 1996.

[31] M.D. Wheeler, Y. Sato, and K. Ikeuchi. Consensus surfaces for modeling $3 \mathrm{~d}$ objects from multiple range images. In Proc. International Conference on Computer Vision, January 1998. 
(A)

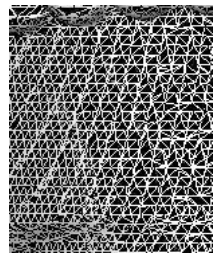

(1)
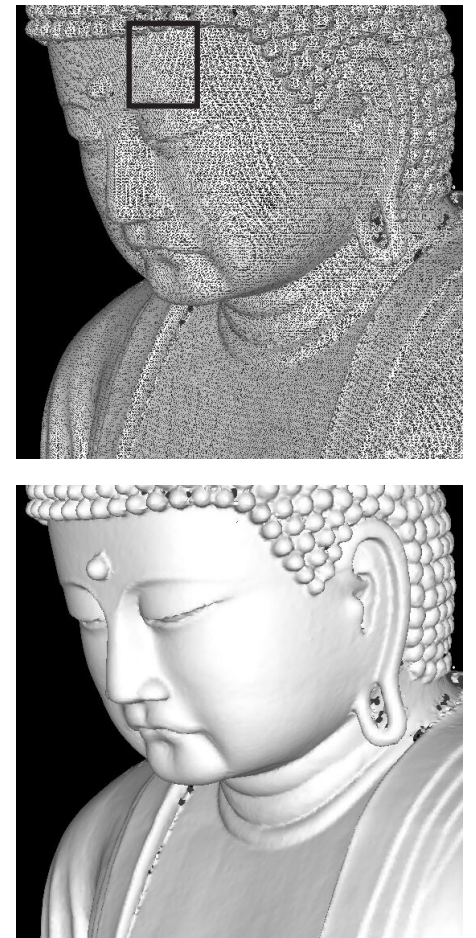

(2)

(3)
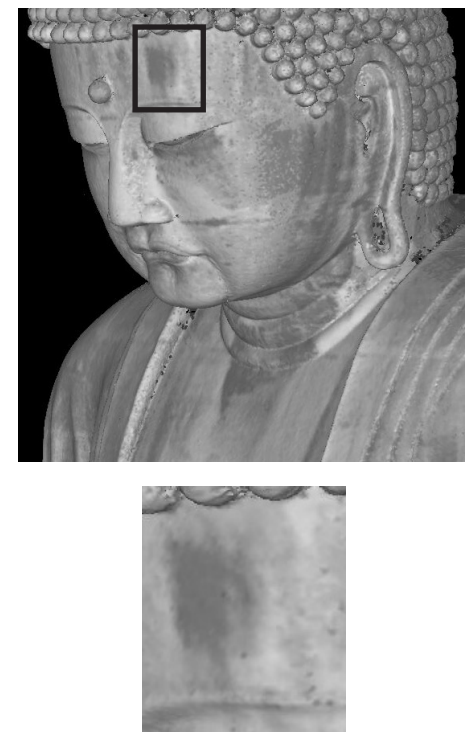

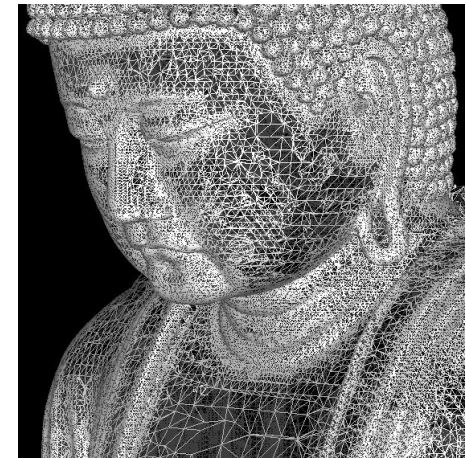

(B)

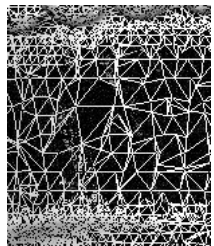

- 10
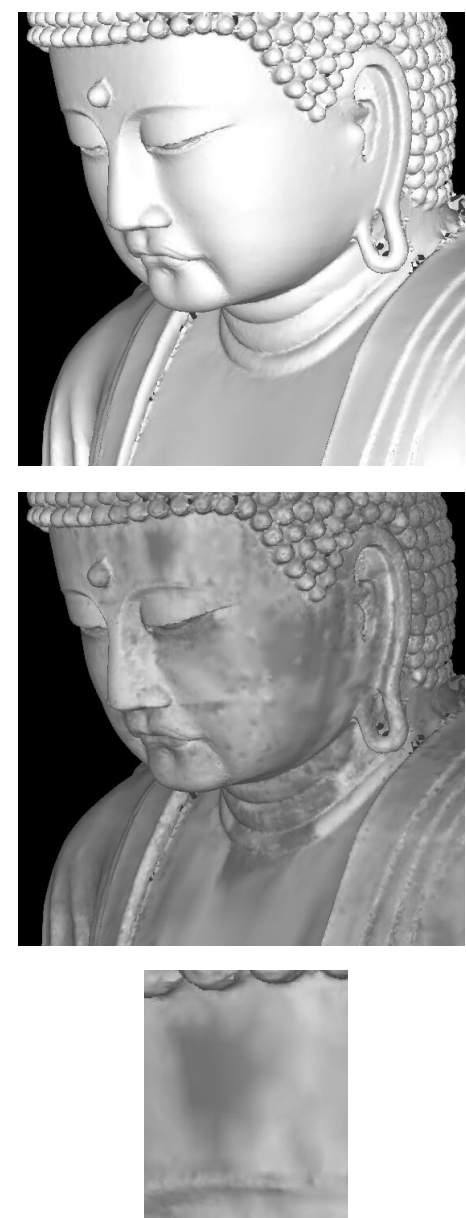

(C)
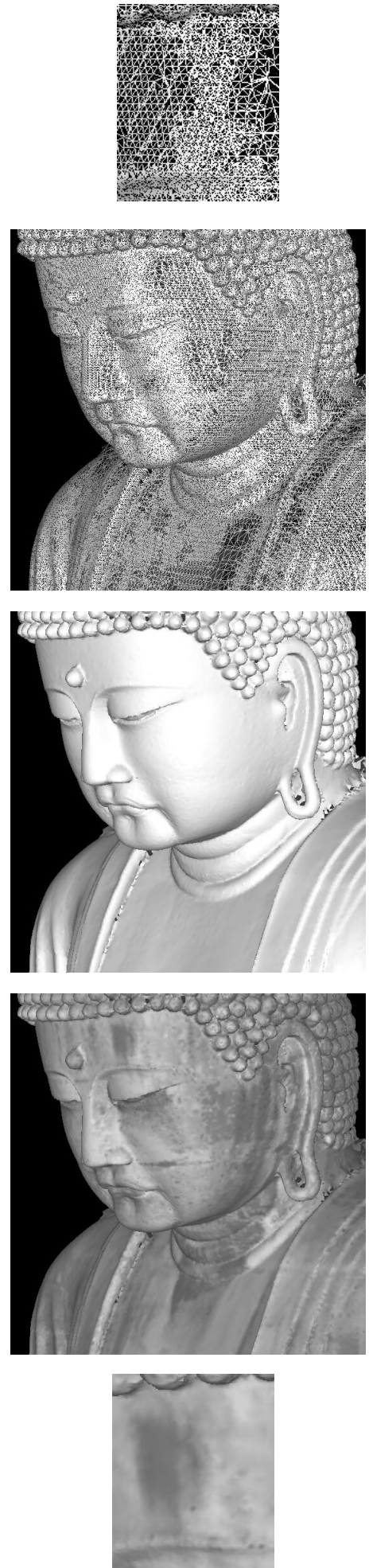

Figure 11: Merging results of the Kamakura Buddha: Column $(\mathrm{A})$ are the models created without adaptive integration. Column (B) are the ones created by adaptive subdivision only based on the curvature of the surface. Column (C) are the ones with adaptive subdivision by the estimation of curvature and LRS. Row (1) are wireframe representations and row (2) are polygonal representations of these models. Row (3) are the images rendered with LRS values. The far upper and far lower rows are zoom-ups of the forehead of the Buddha. 